RADIOTHERAPY

\section{Two-pronged attack}

It is generally accepted that ionizing radiation kills tumours by directly acting on tumour stem cells, but it now seems that this is only part of the story. Richard Kolesnick, Zvi Fuks and colleagues report in Science that radiotherapy also targets the endothelial cells in the microvasculature within growing tumours. The endothelium synthesizes 20 times more acid sphingomyelinase (ASMase) than any other cell in the body and the authors also show that the sphingomyelin apoptotic pathway is integrally involved in the response of endothelial cells to radiation.

They implanted fibrosarcoma or melanoma cells into asmase $e^{+++}$and asmase $^{-1-}$ mice and found that without ASMase tumours grew much faster and endothelial-cell apoptosis was decreased. So, how do these mice respond to irradiation with 10-20 Gy - doses that are equivalent in their biological effects to those given to patients in daily fractionated radiotherapy regimens? When treated with $15 \mathrm{~Gy}, 50 \%$ of fibrosarcomas in the asmase $^{+/+}$mice were cured, whereas there was no tumour control in the asmase $^{-1-}$ mice. The tumour response in the asmase ${ }^{+/+}$mice was preceded by a marked increase in endothelial-cell apoptosis in the first 6 hours after irradiation. This event was lacking in asmase $^{-1-}$ mice.

The authors proved that the resistance of asmase ${ }^{-l-}$ endothelium irradiation with $20 \mathrm{~Gy}$. to radiotherapy. endothelial cell apop

Richard Kolesnick's lab: 11314.cfm to radiation-induced apoptosis was due to direct loss of ASMase within the endothelium by purifying tumour endothelial cells from the mice and subjecting them to irradiation - the response of endothelial cells from asmase ${ }^{-/-}$or asmase a $^{+/+}$ mice mirrored the in vivo responses.

One unexpected finding was that, whereas the responses described hold true for doses of less than $20 \mathrm{~Gy}$, giving doses of $20 \mathrm{~Gy}$ or more activated an alternative tumour response in the asmase $^{-1-}$ mice. Mice null for asmase lacked endothelial-cell apoptosis, even with $20 \mathrm{~Gy}$, but tumours were only resistant at $15 \mathrm{~Gy}$, as a tumour response resulting in $80 \%$ volume reduction was seen within 1 week of

So, if the endothelium remains intact following irradiation, it protects tumour cells from low-dose radiation-induced death. The authors propose that tumours might regulate the radioresponsiveness of their own microvascular endothelium and that developing a strategy to manipulate levels of ASMase might be a promising approach to sensitize tumour cells

Ezzie Hutchinson

(2) References and links ORIGINAL RESEARCH PAPER Garcia-Barros, M. et al. Tumor response to radiotherapy regulated by endothelial cell apoptosis. Science $\mathbf{3 0 0}$

http://www.sloankettering.org/mskcc/html/

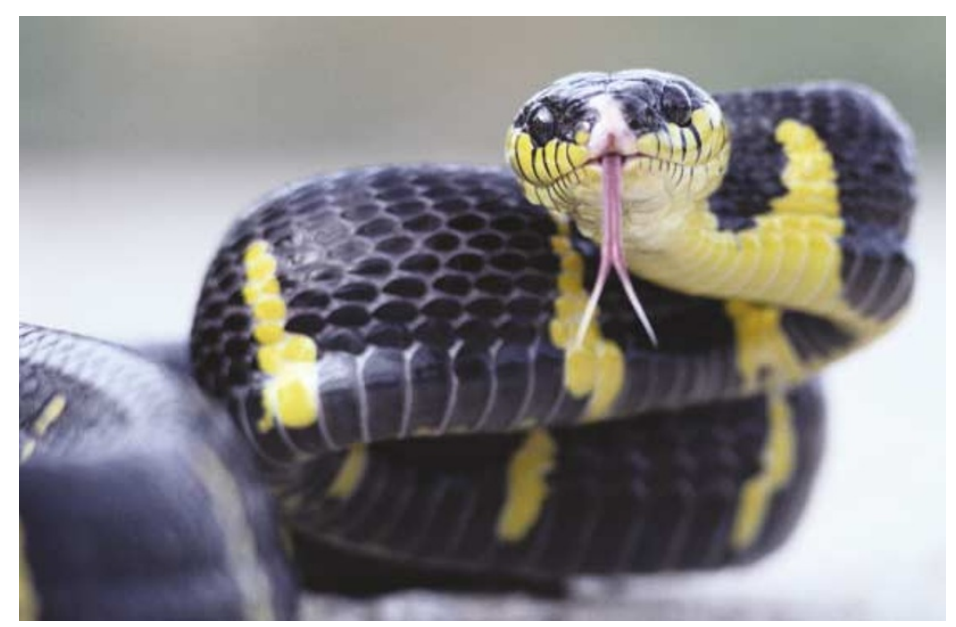

\section{TRIAL WATCH}

\section{Success at last}

The angiogenesis inhibitor bevacizumab (Avastin) lengthens the average survival times for patients with metastatic colorectal cancer by almost 5 months - this is the first time that targeted anti-angiogenic therapy has shown a survival benefit in clinical trials. The results of the Phase III trial, which compared treatment with standard therapy alone or in combination with bevacizumab, were presented by Herbert Hurwitz (Duke University, USA) in a late-breaking abstract at the annual American Society of Clinical Oncology meeting in Chicago. Genentech's bevacizumab is a recombinant humanized monoclonal antibody that binds vascular endothelial growth factor - a key regulator of tumour-induced angiogenesis - and should therefore interfere with tumour growth and metastasis. The trial included more than 800 previously untreated patients who were randomized to receive either the standard therapy of irinotecan, fluorouracil and leucovorin (IFL) in combination with placebo, or with bevacizumab. Hurwitz reported that the median survival time of the patients that received IFL alone was 15.6 months, whereas patients that received bevacizumab plus IFL survived a median of 20.3 months. Cancer did not progress for a median of 10.6 months in the bevacizumab group, compared with 6.2 months in the control group. Tumours also decreased in size by $50 \%$ or more in $45 \%$ of patients who received bevacizumab, but in only $35 \%$ of patients who received IFL alone.

Bevacizumab therapy was well tolerated. Its main side effect was high blood pressure ( $11 \%$ of patients treated with the combination had grade 3 hypertension, versus $2.3 \%$ of the patients that received IFL alone), but this could be easily managed. Phase II trials had indicated that proteinuria and thrombosis might be associated with use of bevacizumab, but these were not supported by the Phase III data. In addition, a rare complication of bevacizumab therapy was perforation of the gastrointestinal tract, leading to one death and the discontinuation of five other patients from the study. Several other Phase III cancer trials to evaluate bevacizumab in patients with metastatic colorectal cancer are underway. The National Cancer Institute is planning to evaluate bevacizumab in patients who are not good candidates for irinotecan as first-line treatment, or who have failed standard therapy with oxaliplatin or irinotecan regimens. Patients in this trial will receive bevacizumab in combination with 5-fluorouracil (5-FU) and leucovorin.

Previous studies have shown that bevacizumab is not effective as a single agent in treating patients with progressive colorectal cancer. Genentech is providing bevacizumab for a large Phase II programme that encompasses more than 22 trials in a variety of malignancies. The drug has shown particular promise in treating patients with metastatic renal-cell cancer - two Phase III trials are opening later this year that will hopefully confirm the positive data observed in a Phase II setting.

Two other new agents, oxaliplatin and cetuximab (Erbitux) have also recently been shown to be effective in randomized trials for patients with advanced and metastatic colorectal cancer. New trials are being planned to compare these two agents and bevacizumab in various combinations. 